TPI-MINN-01/37

July 2001

\title{
Search for Tracker Potentials in Quintessence Theory
}

\author{
Vinod B. Johri* \\ Theoretical Physics Institute, School of Physics and Astronomy, \\ University of Minnesota, Minneapolis, MN 55455, USA
}

\begin{abstract}
We report a significant finding in Quintessence theory that the the scalar fields with tracker potentials have a model-independent scaling behaviour in the expanding universe. So far widely discussed exponential,power law or hyperbolic potentials can simply mimic the tracking behaviour over a limited range of redshift. In the small redshift range where the variation of the tracking parameter $\epsilon$ may be taken to be negligible, the differential equation of generic potentials leads to hyperbolic sine and hyperbolic cosine potentials which may approximate tracker field in the present day universe. We have plotted the variation of tracker potential and the equation of state of the tracker field as function of the redshift $z$ for the model-independent relation derived from tracker field theory; we have also plotted the variation of $V(\Phi)$ in terms of the scalar field $\Phi$ for the chosen hyperbolic cosine function and have compared with the curves obtained by reconstruction of $V(\phi)$ from the real observational data from the supernovae. .
\end{abstract}

PACS numbers: 98.80.Cq, 98.80Es

Emails:vinodjohri@hotmail.com 
The recent measurements [1, 2] of redshift and luminosity distance relation of type Ia supernovae strongly suggest that the cosmic expansion is accelerating. This provides an indirect evidence of the existence of an exotic matter, with positive energy density and negative pressure, dominant in the present day universe. This exotic matter, termed quintessence, behaves like vacuum field energy and has repulsive (anti-gravitational) character due to its negative pressure. The dominance of the quintessence at the present epoch might very well account for the observed acceleration in the cosmic expansion. In fact, any physical phenomenon which generates negative pressure during cosmic evolution may give rise to quintessence. Some of the viable candidates for quintessence are as follows:

i) scalar fields with time varying equation of state are the most favoured for quintessence. Such fields acquire negative pressure during slow roll down of the scalar potential and have been widely discussed [3]-[0] in the literature.

ii) Prigogine-type cosmological models [8]-12] with matter creation out of gravitational energy. The universe is regarded as an open thermodynamical system wherein transformation of gravitational energy into matter induces negative pressure which supports expansion and might cause acceleration in cosmic expansion.

iii) cosmological models with dissipative phenomena where bulk viscosity comes into play.It is well known that the bulk viscosity can give rise to negative pressure in the standard FRW model [13]-15]. without affecting the isotropy of the universe. It is likely that the self-interaction of the cold dark matter in the galactic halos may cause bulk viscosity [27] which might induce cosmic acceleration in the observable universe. Quintessence models with bulk viscosity have been considered by [25, 26].

Long before the supernova observations, Ratra and Peebles [19], and Wetterich [20] had discussed the importance of the rolling scalar fields in the evolution of the universe.The interest in scalar fields was revived with the luminosity distance - redshift observations of type $I_{a}$ supernovae which suggest that about $70 \%$ of the energy content of the universe consists of an exotic matter which induces acceleration in the cosmic expansion. Caldwell et al [3] discussed the possibilty that the scalar fields with evolving equation of state might constitute the exotic matter which counters gravitational attraction and supports expansion of the universe. But for the scalar energy density $\rho_{\phi}$ to be comparable with the matter energy density $\rho_{n}$ of the universe today, the initial conditions for the scalar fields must be set up carefully and fine-tuned. To get rid of the fine-tuning and the coincidence problems, the notion of tracker fields [16, 17] was introduced. It permits the scalar fields with a wide range of initial values of $\rho_{\phi}$ to rolldown along a common evolutionary track with $\rho_{n}$ to end 
up in the observable universe. But simply synchronized scaling of $\rho_{\phi}$ with $\rho_{n}$ is not enough. A realistic tracking behaviour must regulate the growth of $\Omega_{\phi}$ so that the additional contribution of the scalar field to the energy density of the universe does not affect the optimum Hubble expansion which yields the observed Helium abundance at nucleo-synthesis epoch. Again it should not interfere with the process of the formation of galactic structure in the universe and must ensure transition from the matter to scalar field dominated era at the right epoch. To make quintessence physically viable, it should be linked to the cosmological observations. With this objective, the notion of 'integrated tracking' was introduced [18] which essentially implies tracking compatible with astrophysical constraints. We shall briefly review the theory already discussed in [18] to show that the tracker potentials (independent of any particular choice) follow a definite path of evolution, in compatibility with the observational costraints in the physical universe. The scientists have, so far proposed various scalar potentials with tracking properties, mostly in the exponential, power law and hyperbolic forms but they represent the desired behaviour of tracking over a limited range of redshift. In order to compare the model-independent tracking behaviour, predicted by our theory, with the observational results based on SNe meaurements, we assume a spatially flat universe with $\Omega_{n}=0.3$ at the present epoch. It follows from the tracker field theory that the transition to scalar field dominated $\operatorname{era}\left(\Omega_{n} \simeq 0.5\right)$ corresponds to the value of tracking parameter $\epsilon=0.666$ and it takes place at $z=0.526$. We use interpolation techniques to calculate the values of $\Omega_{\phi}$ and $\epsilon$ at different redshifts during cosmic evolution and find that $\epsilon \simeq 0.98$ at the present epoch

Dynamics of Rolling Scalar Fields - Let us consider cold dark matter cosmology with quintessence in the form of rolling scalar fields, with evolving equation of state, which acquire repulsive character (owing to negative pressure) during the late evolution of the universe. Such scalar fields would behave like $\Lambda_{\text {eff }}$ in the present day observable universe, and may turn out to be the most likely form of dark energy which induces acceleration in the cosmic expansion.

Consider the homogeneous scalar field $\phi(t)$ which interacts with matter only through gravity. The energy density $\rho_{\phi}$ and the pressure $p_{\phi}$ of the field are given by

$$
\begin{aligned}
& \rho_{\phi}=\frac{1}{2} \dot{\phi}^{2}+V(\phi) \\
& p_{\phi}=\frac{1}{2} \dot{\phi}^{2}-V(\phi)
\end{aligned}
$$


The equation of motion of the scalar field

$$
\ddot{\phi}+3 H \dot{\phi}+V^{\prime}(\phi)=0, \quad V^{\prime}(\phi) \equiv \frac{d V}{d \phi}
$$

leads to the energy conservation equation

$$
\dot{\rho}_{\phi}+3 H\left(1+w_{\phi}\right) \rho_{\phi}=0
$$

where $w_{\phi} \equiv \frac{p_{\phi}}{\rho_{\phi}}$ and $H \equiv \frac{\dot{a}}{a}$ is the Hubble constant. Accordingly, $\rho_{\phi}$ scales down as

$$
\rho_{\phi} \sim a^{-3\left(1+w_{\phi}\right)}, \quad-1 \leq w_{\phi} \leq 1
$$

Obviously, the scaling of $\rho_{\phi}$ gets slower as the potential energy $V(\phi)$ starts dominating over the kinetic energy $\frac{1}{2} \dot{\phi}^{2}$ of the scalar field and $w_{\phi}$ turns negative.

Since there is minimal coupling of the scalar field with matter/radiation, it follows from Eq.(4) that the energy of matter / radiation is conserved separately as

$$
\dot{\rho}_{n}+3 H\left(1+w_{n}\right) \rho_{n}=0
$$

Accordingly

$$
\rho_{n} \sim a^{-3\left(1+w_{n}\right)}
$$

where $\rho_{n}$ is the energy density of the dominant constituent (matter or radiation) in the universe with the equation of state $p_{n}=w_{n} \rho_{n}$ where $w_{n}=\frac{1}{3}$ for radiation and $w_{n}=0$ for matter.

Although, the scalar field is non-interactive with matter, it affects the dynamics of cosmic expansion through the Einstein field equations. Assuming large scale spatial homogeneity and isotropy of the universe, the field equations for a flat Friedmann model are

$$
H^{2}=\frac{\rho_{n}+\rho_{\phi}}{3 m_{p}^{2}}
$$

and

$$
\frac{2 \ddot{a}}{a}=-\frac{\rho_{n}+\rho_{\phi}+3 p_{n}+3 p_{\phi}}{3 m_{p}^{2}}
$$

where $m_{p}=2.4 \times 10^{18} \mathrm{GeV}$ is the reduced Planck mass.

Denoting the fractional density of the scalar field by $\Omega_{\phi} \equiv \frac{\rho_{\phi}}{\rho_{n}+\rho_{\phi}}$ and that of the matter/radiation field by $\Omega_{n} \equiv \frac{\rho_{n}}{\rho_{n}+\rho_{\phi}}$, equations (8) and (9) may be rewritten as

$$
\Omega_{n}+\Omega_{\phi}=1
$$


and

$$
2 \frac{\ddot{a}}{a}=-\frac{\rho_{n}}{3 m_{p}^{2}}\left[\left(1+3 w_{n}\right)+\left(1+3 w_{\phi}\right) \frac{\Omega_{\phi}}{\Omega_{n}}\right]
$$

The relative growth of $\Omega_{\phi}$ during the cosmic evolution is given by

$$
\frac{\Omega_{\phi}}{\Omega_{n}}=\frac{\Omega_{\phi}^{0}}{\Omega_{n}^{0}}\left(\frac{a}{a_{0}}\right)^{3 \epsilon}
$$

where the tracking parameter $\epsilon \equiv w_{n}-w_{\phi}$ and $\Omega_{\phi}^{0}, \Omega_{n}^{0}$ denote the values of $\Omega_{\phi}$ and $\Omega_{n}$ at the present epoch $\left(a=a_{0}\right)$. As indicated by the recent supernovae observations,$\Omega_{\phi}^{0} \simeq \frac{7}{3} \Omega_{n}^{0}$ at the present epoch; consequently Eq.(12) may be expressed in terms of the red-shift $z$ as below

$$
\left(\Omega_{\phi}^{-1}-1\right)=0.43(1+z)^{3 \epsilon}
$$

If we insist that the scalar field, regardless of its initial value, should behave like $\Lambda_{\text {eff }}$ today, it must obey tracking conditions [16, 17, 18 which have wide ramifications for quintessence fields as already discussed in detail [16, 18]. In nutshell, tracking consists in synchronised scaling of $\rho_{\phi}$ and $\rho_{n}$ along a common evolutionary track with $w_{\phi}<w_{n}$ so as to ensure the restricted growth of $\Omega_{\phi}$ during the cosmic evolution in accordance with the observational constraints.

Search for Tracker Potential - We have already discussed [18] the theory of integrated tracking in brief and shown that the scalar fields can give rise to quintessence $\left(w_{\phi}<0\right.$ ) during the slow rolldown of scalar potential when the kinetic energy term is very small compared to $V(\phi)$. Then from Eq.(1) and (2)

$$
\frac{\dot{\phi}^{2}}{\rho_{\phi}}=1+w_{\phi}
$$

and

$$
V(\phi)=\left(\frac{1-w_{\phi}}{2}\right) \rho_{\phi} \sim \rho_{\phi}
$$

Thus $V(\phi)$ scales down effectively as $\rho_{\phi}$ during tracking. Differentiating Eq.(14) logarithmically with respect to time, we get the slow roll down condition for the scalar potential under the assumption that $\dot{w}_{\phi} \simeq 0$

$$
\pm \frac{V^{\prime}(\phi)}{V(\phi)}=\frac{3 H\left(1+w_{\phi}\right)}{\dot{\phi}}=\sqrt{\frac{3\left(1+w_{\phi}\right)}{\Omega_{\phi} m_{p}^{2}}}
$$

Again differentiating logarithmically and writing $\zeta$ for $\frac{V^{\prime}}{V}$, we get 


$$
\mp \frac{3 H\left(1+w_{\phi}\right) \zeta^{\prime}}{\zeta^{2}}=\frac{\dot{\Omega}_{\phi}}{2 \Omega_{\phi}}
$$

Logaithmic differentiation of Eq.(12) with respect to time yields

$$
\frac{\dot{\Omega}_{\phi}}{\Omega_{\phi}\left(1-\Omega_{\phi}\right)}=3(\dot{\epsilon} \ln a+\epsilon H)
$$

Combining Eq.(17) with Eq. (18) and inserting the value of $\Omega_{\phi}$ from Eq.(16), we get

$$
\mp \frac{\zeta^{\prime}}{\zeta^{2}-k^{2}}=\frac{\dot{\epsilon} \ln a+\epsilon H}{2 H\left(1+w_{\phi}\right)}
$$

Using the criterion $\dot{\epsilon} \geq 0$ for tracking [18], the generic tracker potentials are given by the differential equation

$$
\mp \frac{\zeta^{\prime}}{\zeta^{2}-k^{2}} \geq \frac{\epsilon}{2\left(1+w_{n}-\epsilon\right)}
$$

where $+\zeta^{\prime}$ corresponds to decreasing potentials and $-\zeta^{\prime}$ to increasing potentials and $k^{2}=3\left(1+w_{\phi}\right) / m_{p}^{2}$ According to our notation, the prime denotes differentiation with respect to $\phi$ and an overdot denotes time-derivative.

The dynamics of tracking depends sensitively on the variation of the tracking parameter $\epsilon$ during cosmic evolution. For this reason, integrated tracking links $\epsilon$ to the observational constraints [18. For example, the choice of $\epsilon=0.666$ at $z=0.526$ corresponds to $\Omega_{\phi} \simeq 0.5$ which ensures the onset of acceleration in cosmic expansion at this epoch. Again, $\epsilon \leq 0.035$ at $z=10^{10}$ corresponds to $\Omega_{\phi}<0.14$ which ensures that the observed helium abundance by nuleosynthesis, as successfully predicted by the standard model, is not disturbed by the presence of the scalar field. It is noteworthy that the value of the redshift marking the onset of acceleration depends upon the observed value of $\Omega_{n}^{0}$. The transition to scalar dominated era will occur at $z=0.414$ for $\Omega_{n}^{0}=0.35$ and at $z=0.732$ for $\Omega_{n}^{0}=0.25$ By using interpolation techniques [21], the complete tracking profile of the scalar fields can be mapped for any range of redshift. In particular for the small redshift range, where the variation in the tracking parameter $\epsilon$ is negligible, two exact solutions of Eq.(20) may be obtained as follows:

Case I. Tracker Field with Decreasing Potential

Taking + ve sign with $\zeta^{\prime}, \mathrm{Eq},(20)$ gives on integration, 


$$
\frac{V^{\prime}}{V} \equiv \zeta=-k \operatorname{coth} k\left(\frac{\phi}{\alpha}+\beta\right)
$$

which yields on further integration

$$
V=A \sinh k\left(\frac{\phi}{\alpha}+\beta\right)^{-\alpha}
$$

where $\alpha=\frac{\epsilon}{2(1-\epsilon)}$ and $w_{n}=0$ in the small redshift range under consideration.

This is the analytical derivation of the scalar potential proposed by Urena-Lopez and Matos [22 for tracker fields but it holds good for constant tracking parameter.

Case II. Tracker Field with Increasing Potential

Taking -ve sign with $\zeta^{\prime}$, Eq.(20) gives on integration,

$$
\frac{V^{\prime}}{V} \equiv \zeta=k\left[\tanh k\left(\frac{\phi}{\alpha}-\beta\right)\right]
$$

which yields the integral solution

$$
V=A\left[\cosh k\left(\frac{\phi}{\alpha}-\beta\right)\right]^{\alpha}
$$

The tracker potential (24) is of the same form as proposed by Sahni and Wang [23]. We shall be using it for comparison with the reconstructed potential from the observational data [24].

Comparison with Observational Data - First we use Eq.(15) to show that the tracker potential $V(\phi)$ may be expressed as a function of redshift, in conjunction with Eqs.(5),(7) and (13), as

$$
V(z)=\left(\frac{1+\epsilon-w_{n}}{2}\right)(1+z)^{3(1-\epsilon)} \rho_{\phi}^{0}
$$

in the model independent form where $\epsilon$ conforms to astrophysical constraints and its value at different redshifts is known to us by interpolation [21]. Accordingly, the $V(z)$ curve plotted from Eq.(25) is the characteristic curve for all tracker potentials. It leads to a significant result in Quintessence theory that the tracker potentials scale down along a definite path in the expanding universe independent of the functional form of $V(\phi)$.

Throughout the matter dominated and scalar field dominated era, $w_{n}=0$ and $\epsilon=-w_{\phi}$. With this simplification, we can plot the $V(z)$ curve by writing Eq.(25) in the form 


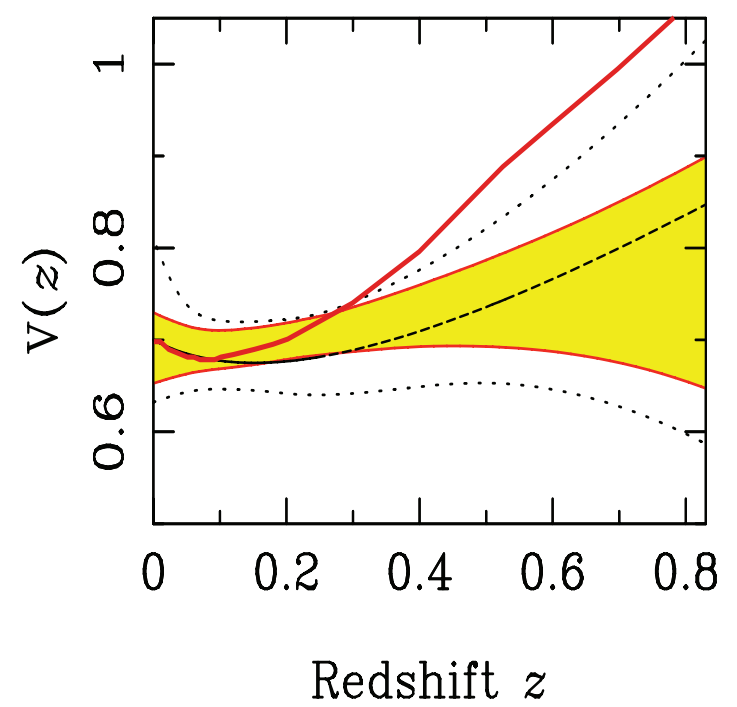

Figure 1: The tracker potential $V(z)$, shown in units of the critical density $\rho_{c r}^{0}$, is plotted as a function of the redshift. The solid line shows the potential from tracker theory and the thin blue line shows the potential reconstructed from the observational data

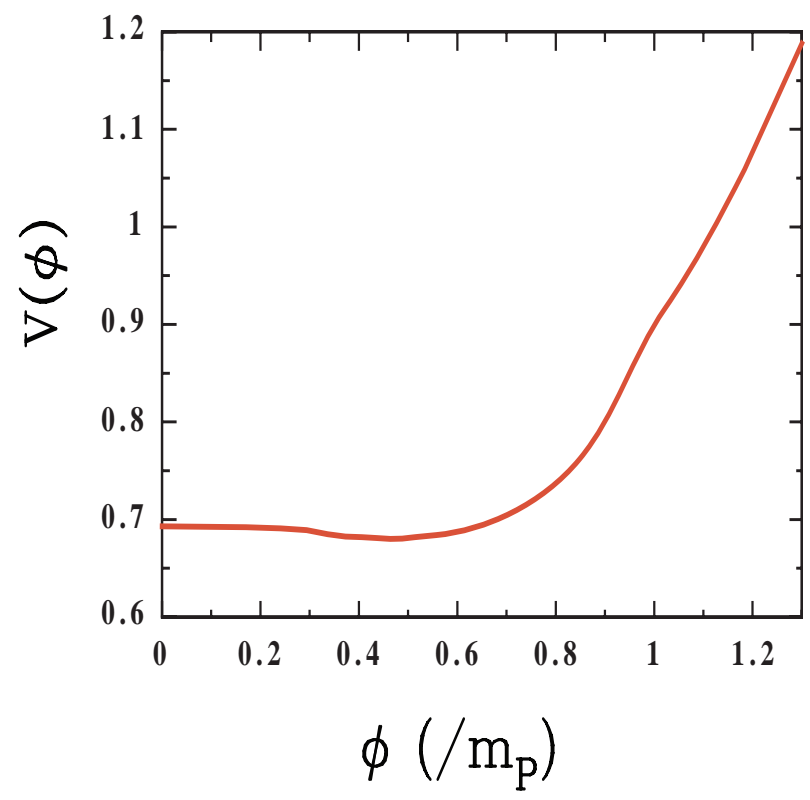

Figure 2: The tracker potential $V(\Phi)$ is shown in units of the critical density $\rho_{c r}^{0}$ at the present epoch. The value of $\Phi$ is shown in units of planck mass. 


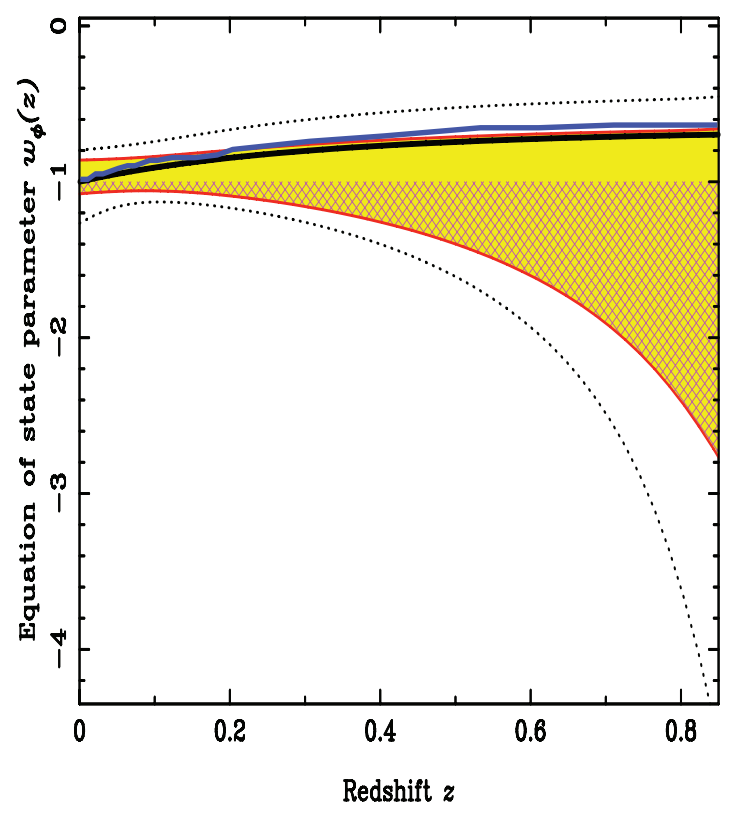

Figure 3: The equation of state parameter $w_{\phi}(z)=\frac{p_{\phi}}{\rho_{\phi}}$ as a function of the redshift $z$. The solid blue line depicts the variation according to tracker field theory, the black line shows the variation corresponding to the reconstructed equation of state from the observational data.

$$
\frac{V(z)}{\rho_{c r}^{0}}=0.35(1+\epsilon)(1+z)^{3(1-\epsilon)}
$$

where $\rho_{c r}^{0}$ is the critical density of the universe at the present epoch. The plotted curve is shown in figure 1.

Let us now examine as to how far the the potential function (24) agrees with the form of $V(\phi)$ reconstructed by Saini et al [24] from the real observational data of the supernovae [1, 2]. We can express potential in units of the critical density and $\Phi$ in units of planck mass and rewrite Eq. (24) in the form

$$
\frac{V(\Phi)}{\rho_{c r}^{0}}=0.35(1+\epsilon)\left[\cosh \frac{\Phi}{m_{p}}\right]^{\alpha}
$$

taking $\Phi=0$ when $z=0$ (since in case of increasing potential $\left(\frac{d z}{d \Phi}>0\right)$ where $\frac{\Phi}{m_{p}} \equiv$ $k\left(\frac{\phi}{\alpha}-\beta\right)$. The $V(\Phi)$ curve(figure 2 ) has been be plotted from Eq.(27) by calculating $\frac{\Phi}{m_{p}}$ in terms of the redshift from the relation 


$$
\cosh \left(\frac{\Phi}{m_{p}}\right)=(1+z)^{3 \epsilon / 2}
$$

The $w_{\phi}(z)$ curve (fig.3) is plotted from the interpolated values of $\epsilon$ for different values of $z$.

Although the variation of $w_{\phi}$ as a function of the redshift $z$, predicted from the tracker field theory, lies within $1 \sigma$ range when compared with the corrersponding curve obtained by Saini et al [24], the variation of the tracker potential $\mathrm{V}$ as a function of $z$ and as a function of $\phi$ appears to have some deviation in the rolldown behaviour. We should like to point out that the reconstruction of $V(\phi)$ from the observational data by Saini et al [24] and our theoretical results derived from tracker field theory both are based on the assumption $\Omega_{n}^{0}=0.3$. This presumption leads to the interpolated value $w_{\phi}=-0.98$ at the present epoch.It implies that the present value of the scalar field energy density is very close to the cosmological constant $\Lambda$ with $\left(w_{\phi}=-1\right)$ and the future expansion of the universe will be given by the scale factor $a \sim \sinh ^{\frac{2}{3}}\left(\frac{3}{2} \sqrt{\Lambda / 3} c t\right)$ However, the initial choice of $\Omega_{n}^{0}=0.33$ leads to $w_{\phi}=-0.77$ at the present epoch and a different tracking path for the tracker potential but it remains model-independent as given by Eq.(25) . The matching of our model with the reconstructed $V(\phi)$ lends support to the view that the scalars fields might be the ultimate choice for the quintessence energy.

Acknowledgments This work was supported by UGC grant from India. The author acknowledges useful discussions and valuable help of Keith Olive, Panagiota Kanti and Tonnis Ter Veldhuis and hospitality of Theoretical Physics Institute, University of Minnesota.

* Permanent Address: Department of Mathematics and Astronomy, Lucknow University, Lucknow 226007. India.

\section{References}

[1] S. Perlmutter et al, Bull.Am.Astron.Soc.29,1351 (1997); ibid,Astrophysics J.,517,565 (1999)

[2] A.G. Riess et al, Astron. J. 116, 1009 (1998);astro-ph/0104455 v1

P. Garnavich et al., Astrophys. J. 493, L53 (1998);

B.P. Schmidt et al., ibid 507, 46 (1998).

[3] R.R.Caldwell,R.Dave and P.J.Steinhardt,Phys.Rev.Lett801582(1998) 
[4] P.G.Ferreira and M.Joyce,Phys.Rev.D58,023503(1998)

[5] D.Wands, E.J.Copeland and A.R.Liddle,Phys.Rev.D.57,4686(1998)

[6] A.R.Liddle and R.J.Scherrer, Phys.Rev.D.59,023509(1999)

[7] S.Dodleson, M.Kaplinghat and E.Stewart, Phys.Rev.Lett 85,5276(2000)

[8] I.Prigogine and J.Geheniau, Proc.Nat. Acad.Sci(USA),93,6245(1986)

[9] I.Prigogine,J.Geheniau,E.Gunzig and P.Nardone,Proc. Nat.Acad.Sci(USA) $85,748(1988)$

[10] V.B.Johri and K.Desikan, Jour.Math.and Physical Sci.25,563(1991)

[11] V.B.Johri and K.Desikan, Jour.Ind Math.Soc.61,42 (1995),Astrophys.Lett and Commun. 33, 287 (1996)

[12] V.B.Johri and S.K.Pandey, Int. Jour. Theor. Phys.,38(7),(1999)

[13] T. Padmanabhan and S.M.Chitre, Phys. Lett.A120,433 (1987)

[14] V.B.Johri and R.Sudharsan, Phys.Lett.A132,316(1988);Phys.Lett.A141,257(1989)

[15] J.A.S.Lima et al, Phys.Rev.D.53,4287 (1996), Phys. Rev.D.54,2571(1996)

[16] I.Zlatev,L.Wang and P.J.Steinhardt,Phys. Rev.Lett.82,896(1999)

[17] P.J. Steinhardt et al, Phys. Rev. D 59, 123504 (1999).

[18] V.B.Johri, Phys.Rev.D.63,103504 (2001)

[19] B.Ratra and P.J.E.Peebles, Phys. Rev.D.37,3406(1988)

[20] C.Wetterich, Nucl.Phys.B302,668(1988)

[21] V.B.Johri (to be published);also see astro-ph/0007079 v2

[22] L.A.Urena-Lopez and T.Matos, Phys.Rev.D.62,081302(2000)

[23] V. Sahni and L.Wang, Phys. Rev.D.62, 103517(2000)

[24] T.D.Saini,S.Raychaudhury, V.Sahni and A.A.Starobinsky, Phys. Rev.Lett.85,1162(2000) 
[25] A.A.Sen, S.Sen and S.Sethi,Phys.Rev.D.63,107501(2001)

[26] S.Sen and A.A.Sen, Phys. Rev.D.63, 124006(2001)

[27] D.N.Spergel and P.J.Steinhardt,Phys.Rev.Lett.84,3760(1999);J.P.Ostriker,ibid 84,5258(2000);b.Moore et al, Astrophys.J.Lett.535,L21(2000) 